
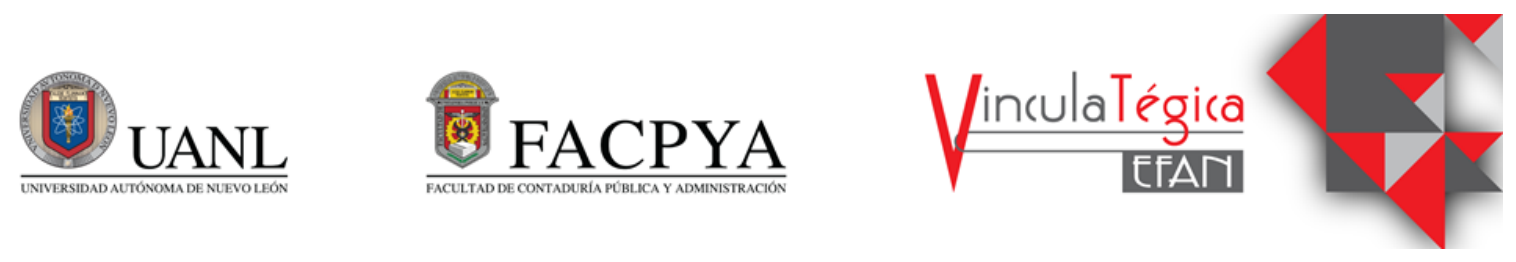

\title{
El número de empleados en el comportamiento tributario de los microempresarios
}

\author{
María del Mar Obregón Angulo ${ }^{1}$, Santiago Alejandro Arellano Zepeda² y Neri José de \\ Jesús Moreno ${ }^{3}$ \\ ${ }^{1}$ Facultad de Ciencias Administrativas y Sociales Universidad Autónoma de Baja California, \\ marymar@uabc.edu.mx, Boulevard de los lagos sin número Fraccionamiento Valle dorad,646-176662. \\ ${ }^{2}$ Facultad de Ciencias Administrativas y Sociales Universidad Autónoma de Baja California, \\ alejandroarellano@uabc.edu.mx, Boulevard de los lagos sin número Fraccionamiento Valle dorad,646- \\ 176662 \\ ${ }^{3}$ Facultad de Ciencias Administrativas y Sociales Universidad Autónoma de Baja California, \\ jjmoreno@uabc.edu.mx, Boulevard de los lagos sin número Fraccionamiento Valle dorad,646-176662
}

Información del artículo revisado por pares

Fecha de aceptación: junio-2021

Fecha de publicación en línea: diciembre-2021

DOI: https://doi.org/10.29105/vtga7.1-152

\section{Resumen}

Esta investigación está dirigida a comparar como afecta el número de empleados que tienen los microempresarios representados como una variable de control, con el comportamiento tributario y los factores que lo afectan que son, la credibilidad, la complejidad, la capacidad económica, la implementación de las TIC, la facturación electrónica, la costumbre, la resistencia al cambio, la carga tributaria y la fiscalización, especialmente con el factor que inciden en este comportamiento de la implementación de las TIC, el cual está directamente ligado con las obligaciones derivadas del pago de la nómina y el correcto cumplimiento de sus obligaciones fiscales. Para tal efecto se realizó una investigación de corte transversal, no experimental que fue descriptiva y explicativa, donde se examinaron los factores que se obtuvieron aplicando el método logic, de carácter ordinal y fueron comparados estos en relación con la variable de control número de empleados, para determinar si esta incidía en los resultados obtenidos. De acuerdo a los resultados del presente estudio se encontró que
Abstract
This research is aimed at comparing how it affects the number of employees that microentrepreneurs have, represented as a control variable, with tax behavior and the factors that affect it, which are credibility, complexity, economic capacity, the implementation of the ICT, electronic invoicing, custom, resistance to change, the tax burden and supervision, especially with the factor that affects this behavior of the implementation of ICT, which is directly linked to the obligations derived from the payment of the payroll and the correct fulfillment of your tax obligations. For this purpose, a nonexperimental, cross-sectional investigation was carried out that was descriptive and explanatory, where the factors obtained by applying the logic method, ordinal in nature, were examined and these were compared in relation to the control variable number of employees, to determine if this affected the results obtained. According to the results of the present study, it was found that within the microentrepreneurs who have employees, the ICT implementation variable is 
dentro de los microempresarios que tienen empleados, la variable de implementación de las TIC es más representativa, en comparación con los resultados cuando esta variable no es tomada en cuenta.

Palabras clave: Comportamiento tributario, Factores, Implementación de las TIC, Numero de Empleados.

\section{INTRODUCCIÓN}

Siempre ha sido de interés de los diferentes gobiernos establecer cuáles son los factores que determinan el comportamiento tributario, por lo que se han llevado a cabo estudios, para poder identificarlos, y saber de qué manera influyen en el incumplimiento de las obligaciones fiscales, principalmente la del pago de los impuestos, así que a realizando una revisión de investigaciones previas y artículos relacionados con el tema, se identifican los factores que están directamente relacionados con el cumplimiento voluntario de las obligaciones fiscales, y, como estos se modifican por alguna circunstancia especial como seria si cuentan con trabajadores o el número de ellos y si este es determinante de alguno de ellos.

Basándonos es el siguiente modelo que se muestra en la figura 1, que se obtuvo de una Tesis Doctoral, es que se decide identificar si la variable de control asignada al número de empleados en las microempresas, influye en alguna de las variables que modifican en el comportamiento tributario de los more representative, compared to the results when this variable is not taken into account.

Keywords: Tax behavior, ICT Implementation, Number of Employees, Factors.

JEL: A10, M15,M21, M41

microempresarios, para determinar si el tenerlos o no modifica la representatividad de los mismos.

Figura 1. Factores del comportamiento tributario que influyen a los microempresarios

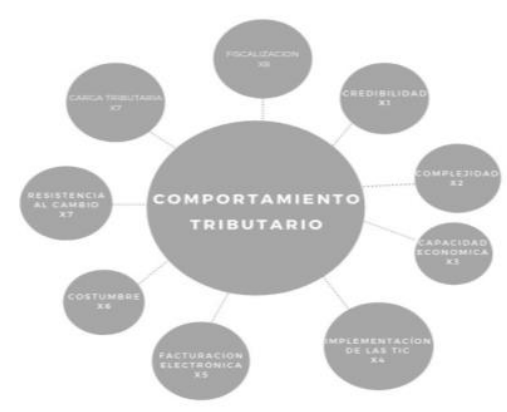

Fuente: Elaboración propia

Este modelo considera que los factores que influyen en el comportamiento de los microempresarios son: la credibilidad, la complejidad, la capacidad económica, la implementación de las TIC, la facturación electrónica, la costumbre, la resistencia al cambio, la carga tributaria y la fiscalización.

Una de las obligaciones que afecta a 
contribuyentes del Régimen de Incorporación Fiscal (RIF), es cumplir con el Comprobante Fiscal Digital por Internet para nómina, la cual es una carga importante, el Código Fiscal de la Federación, establece como obligación para los microempresario que por las retenciones que hagan a sus trabajadores deberán emitir comprobantes digitales, los cuales se deben de elaborar a través de páginas autorizadas por el Sistema de Administración tributaria (SAT) o a través de las aplicaciones que se encuentran disponibles de manera gratuita en los portales oficiales.

También la Ley del Impuesto sobre la renta establece que deberán expedir y entregar comprobantes fiscales a las personas que reciban pagos por concepto de sueldos y asimilados a salarios en la fecha en que los realice, mismos que podrán utilizarse como como comprobante de pago haciendo de constancia o recibo, por la entrega por correo electrónico que se haga del comprobante fiscal digital por internet de las remuneraciones cubiertas a los trabajadores.

Estas obligaciones representan un punto importante de entrada a la formalidad en el aspecto tributario y de seguridad social, así como preparan a los contribuyentes para una eventual inserción al Régimen de Personas con Actividades Empresariales y profesionales que establece el Capitulo $2^{\circ} \mathrm{del}$ Título IV de la Ley del Impuesto Sobre la Renta (López, 2015). En este contexto, los microempresarios que no pudieran cubrir los gastos para adaptarse al Régimen de Incorporación fiscal y al que estarán sujetos en 10 años y que competirán con las grandes empresas, haciendo frente a costos fijos mayores con el riesgo de no poder competir con las grandes empresas y quedar fuera de mercado elevando el poder monopólico u oligopólico, lo cual al final afecta al consumidor.

En México la economía informal representa el $60 \%$ del mercado laboral y al implementar estos controles los costos fijos aumentarían porque éstas tendrían que capacitarse para el uso de las plataformas y la nueva tecnología, contratar personal capacitado o servicios externos para poder cumplir, invertir en equipos; además de contar con servicio de internet al quedar registrados todos sus ingresos pagarían más impuestos ya que no podrían manipular estos como estaban acostumbrados a hacerlo, todo esto, se convertirá en un incentivos para pasarse al sector informal, agravando la situación da el mercado laboral en México. (García 2015).

Al estar sujetos a la Obligación de emitir los comprobantes fiscales digitales por internet dentro del Código Fiscal de la Federación, deberá emitir dichos comprobantes por los pagos y remuneraciones que se haga a los trabajadores derivadas de la nómina, y por los ingresos que se perciban o 
por las retenciones de contribuciones que efectúen a través de la página de Internet del SAT (Gómez, 2017).

Obligaciones derivadas del pago de la nómina:

1- Ley Federal del Trabajo. En esta ley se regula la relación laboral pago de salario, aguinaldo, prima vacacional, prima dominical, días de descanso obligatorios, vacaciones, finiquitos $\mathrm{e}$ indemnizaciones.

2- Ley del Impuesto sobre la renta. calcular, retener y enterar (pagar) mensualmente el impuesto que se genera por el pago de un salario; de igual manera, emitir el recibo de nómina en formato XML, proceso conocido con el término "timbrar".

3- Ley del seguro social dar de alta a sus trabajadores en el IMSS y a pagar las cuotas obrero-patronales a través de aplicaciones como IMSS desde su empresa (IDSE) y Sistema Único de Aportaciones (SUA) así como el uso del escritorio virtual del IMSS.

4- Ley del Instituto Nacional de la vivienda para los trabajadores (INFONAVIT). Pago de aportaciones y en su caso retención y entero de las amortizaciones de crédito de los trabajadores a través del SUA y de la plataforma de portal empresarial.

Todo esto conlleva obligaciones las cuales se encuentran ligadas directamente con el uso e implementación de las TIC por lo que resulta importante conocer como estas están relacionadas con el número de trabajadores que tienen en las microempresas y como afecta la manera que afectan en el comportamiento tributario de las mismas.

\section{MARCO TEÓRICO}

Revisaremos dos conceptos importantes relacionados con las variables de esta investigación que son el comportamiento tributario y la implementación de las TIC.

\subsection{Comportamiento tributario}

El comportamiento tributario se considera la manera en que se da cumplimiento de las obligaciones que tienen los contribuyentes las cuales incluyen las declaraciones, la facturación, pagos, entrega de informes de manera oportuna, reportes financieros, así como todos los anexos y disposiciones que las leyes establezcan (Arias y Torres 2015).

Existen varios factores que pueden determinar el cumplimiento de estas obligaciones, uno de los supuestos seria que las personas al pagar sus impuestos están 
seguras que esto les traerá beneficios directos hacia sus familias y la comunidad, como también podría ser lo contrario de que dejen de pagar o no lo hagan de manera correcta al considerar que no se verán compensados con mejores condiciones de salud, educación o seguridad entre otros, por lo cual se puede estableces que algunos elementos como podrían ser el desconocimiento de los procedimientos, leyes, normas o la conciencia tributaria, así como la complejidad para interpretar o cumplir los mismos tendrían que estar relacionados directamente con el cumplimiento de las obligaciones.

La conciencia tributaria puede ser un determinante al momento de decidir si efectuaremos el pago de nuestras contribuciones o no, es decir que realmente queremos contribuir, así lo señalo Gaona 2019, y por otra parte esta, si tenemos lo necesario para poder contribuir, contando col los mecanismos y herramientas para efectuar el cumplimiento de la contribución "saber y poder contribuir".

El saber cómo contribuir, se refiere a que tenemos los conocimientos necesarios y la información, sobre las reglas y procedimientos, así como el manejo de los programas y plataformas para un correcto cumplimiento y determinación de los procedimientos tributarios. Por eso se considera que la complejidad que tiene el cumplimiento de nuestras obligaciones y la carga tributaria influyen en el cumplimento de las mismas, ya que podría querer cumplir, pero el desconocimiento de las herramientas adecuadas y de la utilización de las TIC ocasionarían no cumplir y contribuir.

A continuación, se presenta en la tabla 1 la relación de diversas investigaciones relacionadas con el comportamiento tributario consultadas.

\section{Tabla 1. Investigaciones relacionadas con el Comportamiento Tributario}

\begin{tabular}{|c|c|}
\hline AUTOR & RESULTADO \\
\hline $\begin{array}{l}\text { Bedoya, A. y } \\
\text { Vásconez, B. } \\
(2010)\end{array}$ & $\begin{array}{l}\text { Introduce la variable 'moral', } \\
\text { como un factor determinante en } \\
\text { la evasión y el cumplimiento } \\
\text { tributario }\end{array}$ \\
\hline Burga (2015) & $\begin{array}{l}\text { El cumplimiento de sus } \\
\text { obligaciones se encuentra } \\
\text { influido por la conciencia } \\
\text { tributaria de las empresas } \\
\text { comerciales. }\end{array}$ \\
\hline Casar (2013) & $\begin{array}{l}\text { Establece cuatro determinantes } \\
\text { para el cumplimiento de las } \\
\text { obligaciones en materia fiscal. }\end{array}$ \\
\hline $\begin{array}{l}\text { Castañeda } \\
(2014)\end{array}$ & $\begin{array}{l}\text { Factor central en el estudio del } \\
\text { grado de moral tributaria es la } \\
\text { corrupción. Definiendo a la } \\
\text { moral como su disposición a } \\
\text { pagar impuestos. } \\
\text { Utilizando varios modelos probit } \\
\text { ordenados, estimados con base } \\
\text { en datos de la encuesta realizada } \\
\text { en } 2010 \text { por Latinobarómetro. }\end{array}$ \\
\hline $\begin{array}{l}\text { Gaibor, A., } \\
\text { Manya, M., } \\
\text { Piedrahita, D., } \\
\text { y Carrera, } \\
\text { W(2010) }\end{array}$ & $\begin{array}{l}\text { Uno de los mayores fenómenos } \\
\text { que afectan el sistema tributario } \\
\text { es la evasión fiscal }\end{array}$ \\
\hline $\begin{array}{l}\text { González } \\
(2006)\end{array}$ & $\begin{array}{l}\text { Los pequeños y medianos } \\
\text { contribuyentes de baja }\end{array}$ \\
\hline
\end{tabular}


significación fiscal son los más difíciles de fiscalizar, ya que en la mayoría de ellos la economía informal o subterránea en una práctica común en los países latinoamericanos.

\begin{tabular}{|l|l|}
$\begin{array}{l}\text { Hinojosa } \\
(2013)\end{array}$ & $\begin{array}{l}\text { Factores externos y psicológicos } \\
\text { para el incumplimiento de las } \\
\text { obligaciones fiscales }\end{array}$ \\
\hline $\begin{array}{l}\text { Lozano } \\
\text { Rodríguez } \\
(2014)\end{array}$ & $\begin{array}{l}\text { Para el cumplimiento de las } \\
\text { obligaciones fiscales por parte de } \\
\text { los microempresarios deben de } \\
\text { establecerse regímenes } \\
\text { preferenciales }\end{array}$ \\
\hline
\end{tabular}

Fuente: elaboración propia con los datos e la bibliografía consultada.

\subsection{Implementación de las TIC}

En México cerca de 60 por ciento de la población económicamente activa (PEA) es informal y no cuenta con seguridad social; el Banco Mundial mostró su preocupación por los alcances del fenómeno y es punto pendiente en su agenda ya que lo considera alarmante. La informalidad es considerada como un síntoma de poco desarrollo y de baja productividad para un país, y es común que esté se asocie al desempleo, el ambulantaje, la falta de tecnología y la carencia de seguridad social, así como con los efectos negativos que ocasiona en la recaudación fiscal.

Por tanto, aquellos que ya pertenecían al mercado laboral formal, y decidieron ya no continuar en el para incorporarse al informal, lo han hecho debido a lo complejo y restrictivo que éste se ha tornado o por no poder invertir en nueva tecnología, o por las sanciones que se establecen por no cumplirlas, lo que muestra una situación compleja y multifacética a la cual se enfrenta el país.

Existen diversos estudios sobre el tema de que es lo que causa la informalidad y coinciden en que algunas de estas serían la educación, la edad, el género, el estado civil y el ingreso. Castells (1989) y Harris y Todaro (1970) señalan, como los motivos prioritarios, lo rígido de las leyes que se generan en el mercado laboral; aspectos como la carencia de tecnología y el bajo nivel educativo motiva su fragmentación y crean uno dual.

A continuación, se muestra la tabla 2 en la cual se relacionan investigaciones relacionadas con esta variable independiente relacionada con la implementación de las TIC.

Tabla 2. Investigaciones relacionadas con la implementación de las TIC

\begin{tabular}{|c|c|}
\hline AUTOR & RESULTADO \\
\hline $\begin{array}{l}\text { Álvarez, } \\
\text { L. (2014) }\end{array}$ & $\begin{array}{l}\text { Micro, pequeñas y medianas } \\
\text { empresas de la región no están } \\
\text { preparadas para cumplir con las } \\
\text { obligaciones fiscales utilizando las } \\
\text { tecnologías de información y } \\
\text { comunicación }\end{array}$ \\
\hline $\begin{array}{l}\text { Castells } \\
1989\end{array}$ & $\begin{array}{l}\text { La falta de Tecnología es un factor } \\
\text { determinante en el cumplimiento de } \\
\text { las obligaciones fiscales. }\end{array}$ \\
\hline $\begin{array}{l}\text { Harris y } \\
\text { Todaro } \\
1970\end{array}$ & $\begin{array}{l}\text { Señalan, como los motivos } \\
\text { prioritarios, aspectos como la } \\
\text { carencia de tecnología y el bajo nivel } \\
\text { educativo. }\end{array}$ \\
\hline
\end{tabular}

Nota: Elaboración Propia a partir de las referencias consultadas. 
Explicativa porque lo sitúa a partir

\section{MÉTODO}

Se llevó a cabo un levantamiento de datos relacionados con los factores que se consideraron influyen en el comportamiento tributario, de los microempresarios para luego analizarlos mediante un estudio descriptivo.

La investigación fue transversal, y posee un enfoque cuantitativo, con alcance exploratorio y correlacional.

Fue llevada a cabo con datos relacionados a los factores que influyen el comportamiento tributario de las microempresas en México.

Para llevarla a cabo se estableció la siguiente Hipótesis: La implementación de las TIC influye en el comportamiento tributario de los microempresarios en relación al número de trabajadores que tienen.

\subsection{Tipo y diseño de la Investigación}

Para cada una de nuestras variables dependientes e independientes el tipo de esta investigación es cuantitativa.

Descriptiva, ya que se analizaron una serie de datos para comprobar que la variable considerada (implementación de las TIC) tienen un efecto en el comportamiento tributario de las microempresas. de un evento determinado que fue la entada en vigor de la reforma fiscal del 2014, y causal porque se busca conocer las razones o causas que provocan ciertas situaciones, hechos o fenómenos.

El diseño de la investigación es no experimental transversal, propone un análisis de los factores determinantes que inciden en el comportamiento tributario de las microempresas, a través de la percepción de los microempresarios.

La variable dependiente de la investigación es Comportamiento Tributario, y la variable independiente en uno de los factores que inciden en el: Implementación de las TIC, en función de la variable de control el número de empleados que tienen.

\subsection{Tamaño y determinación de la muestra}

Para poder definir el tamaño de la muestra, ya que la población objeto de estudio no se puede conocer con exactitud, se procedió a determinar la muestra con base en una población infinita, por la cual se consideró un intervalo de confianza de $95 \%$.

Al realizar los cálculos, se obtuvo un tamaño de muestra de 384 encuestas, utilizando la formula con base en proporciones.

Se aplicaron y la tasa de respuesta fue de 390 encuestas aplicadas que equivale al 
$101.56 \%$ de la muestra calculada, por lo cual se considera apropiada y representativa.

\section{RESULTADOS}

De los datos recolectados se procesaron a través del programa estadístico SPSS Statistics 22 y de Eviews.

Se Integraron dos diferentes bases de datos: La primera se integró por los resultados obtenidos por las variables dependientes e independientes y la segunda base de datos se construyó de los datos obtenidos por las variables de control entre las cuales se estableció como una de ellas el número de trabajadores del encuestado.

La fiabilidad del instrumento aplicado fue comprobada con apoyo de la prueba Alfa de Cronbach. Para cada una de las variables dependientes consideradas que son; credibilidad, costumbre y capacidad económica, según se muestran en la tabla 3. A partir del resultado obtenido el instrumento se considera aceptable.

Tabla 3. Estadístico de Fiabilidad.

$\begin{array}{cc}\text { Alfa de Cronbach } & \text { N de elementos } \\ .885 & 20\end{array}$

También se corroboro la validez de los constructores a través del índice de KMO. Este valor se obtiene haciendo un análisis factorial, a partir de reducción de variables.

\section{Trabajadores promedio.}

La siguiente variable de control que se utilizó es el número de trabajadores que laboran dentro de las microempresas encuestadas donde se establecieron los siguientes rangos:

1- Sin trabajadores

2- 1 a 10 trabajadores

3- De 11 a 30 trabajadores

4- De 31 a 50 trabajadores

5- Más de 50 trabajadores

Se observa en el siguiente gráfico (figura 2) que la mayoría de los microempresarios representando el $40 \%$ no tienen empleados, seguidos por lo que tienen de 0 a 10 trabajadores $38 \%$ y el $15 \%$ que tienes de 11 a 30 . A continuación, observamos la figura 1 en donde se encuentra representados el personal ocupado por las empresas participantes dentro de esta investigación:

Figura 2. Número de trabajadores en las empresas encuestadas

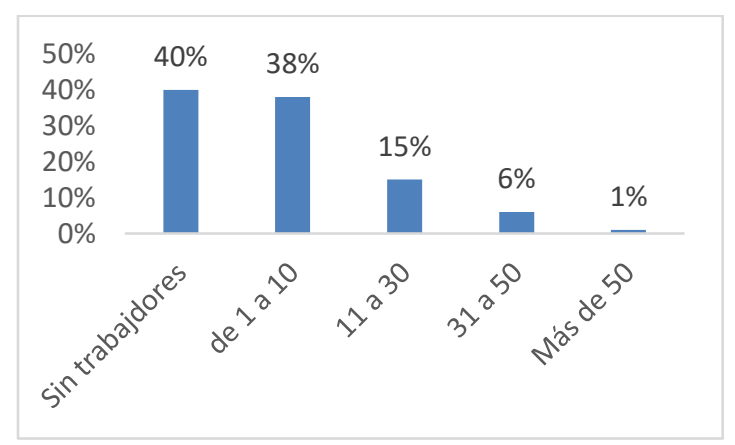


Fuente: elaboración propia

Después de realizar el análisis de los datos obtenidos en las encuestas se obtuvieron los resultados de cuáles eran los factores que más incidían en el comportamiento tributario de los microempresarios encontrando los siguientes resultados que se muestran en la tabla 4.

Tabla 4. Resultados obtenidos sobre factores que inciden en el comportamiento tributario

\begin{tabular}{ll}
\hline NOMBRE & REPRESENTACION \\
\hline
\end{tabular}

$\begin{array}{lc}\text { Resistencia al Cambio } & 10.58 \% \\ \text { Carga Tributaria } & 10.52 \% \\ \text { Complejidad } & 10.18 \% \\ \text { Credibilidad } & 8.87 \% \\ \text { Costumbre } & 7.24 \% \\ \text { Facturación Electrónica } & 6.50 \% \\ \text { Fiscalización } & 5.90 \% \\ \text { Implementación de las TIC } & 4.7 \%\end{array}$

Fuente: Elaboración propia a partir de los resultados de análisis factorial

En estos resultados la implementación

de las TIC es el que tiene menor representatividad de los factores.

Una vez obtenida esta información de las variables independientes se incorporaron los factores de la variable dependiente la cual está representada por el comportamiento tributario de los microempresarios para aplicar nuestros datos el método logit y llevar a cabo los análisis correspondientes.
La utilización de este método permite, además de obtener estimaciones de la probabilidad de un suceso, identificar los factores de riesgo que determinan dichas probabilidades, así como la influencia o peso relativo que éstos tienen sobre las mismas.

A continuación, se presenta la tabla 5 en donde se encuentra el análisis que se llevó a cabo con los factores con el método logit. en el cual al compararlos con la variable dependiente se observa que las cinco primeras variables son significativas al tener su valor de 0.0000. Las cuales representa la credibilidad, complejidad, el uso las tecnologías de Información y la facturación electrónica como factores que inciden en el comportamiento tributario ya que explican el $95 \%$.

Tabla 5. Análisis por el método Logit

\begin{tabular}{cc}
\hline NOMBRE & PROB. \\
\hline Credibilidad & 0 \\
Complejidad & 0 \\
Implementación de las TIC & 0 \\
Facturación Electrónica & 0 \\
Costumbre & 0 \\
Resistencia al cambio & 0.0877 \\
Carga Tributaria & 0.8964 \\
Fiscalización & 0.0611
\end{tabular}

Fuente: Elaboración propia con los datos obtenidos del Eviews, en el Método Logit

Como se puede observar al incluir a la variable dependiente la implementación de las 
TIC resulta ser más significativa, que cuando no se relaciona con la variable dependiente.

Estos resultados obtenidos llevaron a un nuevo análisis para determinar si alguna de las variables de control utilizadas en el estudio tenían alguna incidencia en los resultados, en especial se buscó comprobar si el hecho de tener trabajadores los microempresarios, tiene alguna incidencia en la variable independiente relacionada con la implementación de las TIC.

Para llevar a cabo este análisis se multiplico a cada una de las variables dependiente e independientes por los factores obtenidos en la variable de control para la cual definiremos su nomenclatura de la siguiente manera

$$
\mathrm{Xt} \quad \text { Número de trabajadores }
$$

Por lo cual aplicamos la siguiente formula:

ymed c x $1 \times 1 * x t \times 2 \times 2 * x t \times 4 \times 4 * x t \times 5 \times 5 * x t$ x6 x6*xt x7 x7*xt x8 x8*xt x9 x9*xt

Se obtuvieron los siguientes resultados que se muestran en la tabla 6 .

\section{Tabla 6. Comparación con Variable de} Control

\begin{tabular}{|c|c|c|c|c|}
\hline \multicolumn{5}{|c|}{$\begin{array}{l}\text { Dependent Variable: YMED } \\
\text { Method: ML - Ordered Logit (Quadratic hill climbing) } \\
\text { Date: } 11 / 14 / 19 \text { Time: } 13: 38 \\
\text { Sample: } 1390 \\
\text { Included observations: } 377 \\
\text { Number of ordered indicator values: } 5 \\
\text { Convergence achieved after } 7 \text { iterations } \\
\text { QML (Huber/White) standard errors \& covariance }\end{array}$} \\
\hline Variable & Coefficient & Std. Error & z-Statistic & Prob. \\
\hline $\mathrm{x} 1$ & 0.516994 & 0.280841 & 1.840876 & 0.0656 \\
\hline $\mathrm{X} 1^{\star} \times \mathrm{XT}$ & 0.274687 & 0.204772 & 1.341428 & 0.1798 \\
\hline X2 & 0.620091 & 0.250469 & 2.475724 & 0.0133 \\
\hline $\mathrm{X} 2{ }^{*} \mathrm{XT}$ & 0.104866 & 0.158485 & 0.661676 & 0.5082 \\
\hline $\mathrm{X} 4$ & 1.330036 & 0.464207 & 2.865181 & 0.0042 \\
\hline$X 4^{*} X T$ & -0.355145 & 0.276811 & -1.282989 & 0.1995 \\
\hline X5 & 0.429505 & 0.372281 & 1.153713 & 0.2486 \\
\hline$X 5^{*} \mathrm{XT}$ & 0.734125 & 0.288260 & 2.546749 & 0.0109 \\
\hline X6 & 0.310345 & 0.339274 & 0.914732 & 0.3603 \\
\hline $\mathrm{X} 6{ }^{*} \mathrm{XT}$ & 0.180248 & 0.256044 & 0.703971 & 0.4815 \\
\hline X7 & 0.333732 & 0.223408 & 1.493822 & 0.1352 \\
\hline$X 7^{*} \times T$ & -0.108826 & 0.149577 & -0.727557 & 0.4669 \\
\hline X8 & -0.235444 & 0.283857 & -0.829445 & 0.4069 \\
\hline$X 8^{*} \times T$ & 0.209588 & 0.218995 & 0.957045 & 0.3385 \\
\hline X9 & 0.484815 & 0.254133 & 1.907718 & 0.0564 \\
\hline$X 9 * X T$ & -0.261024 & 0.170464 & -1.531258 & 0.1257 \\
\hline \multicolumn{5}{|c|}{ Limit Points } \\
\hline LIMIT_2:C(17) & -2.570034 & 0.212996 & -12.06609 & 0.0000 \\
\hline LIMIT_3:C(18) & 0.003566 & 0.122581 & 0.029095 & 0.9768 \\
\hline LIMIT_4:C(19) & 1.995940 & 0.181679 & 10.98607 & 0.0000 \\
\hline LIMIT_5:C(20) & 4.482298 & 0.353038 & 12.69637 & 0.0000 \\
\hline Pseudo R-squared & 0.248423 & \multirow{5}{*}{\multicolumn{2}{|c|}{$\begin{array}{l}\text { Akaike info criterion } \\
\text { Log likelihood } \\
\text { Restr. log likelihood } \\
\text { Avg. log likelihood }\end{array}$}} & 2.330985 \\
\hline Schwarz criterion & 2.539592 & & & -419.3907 \\
\hline Hannan-Quinn criter. & 2.413786 & & & -558.0140 \\
\hline LR statistic & 277.2466 & & & -1.112442 \\
\hline Prob(LR statistic) & 0.000000 & & & \\
\hline
\end{tabular}

Fuente: Datos obtenidos de Eviews al llevar a cabo el análisis.

En esta tabla se muestra los resultados obtenidos al relacionar el número de trabajadores con las variables que inciden con el comportamiento tributario.

Así, al realizar la comparación de los factores con la variable de control trabajadores la cual representa número de trabajadores que laboran en la empresa se observa que el factor Implementación de las TIC es significativo ya que al tener más trabajadores se les complican el cumplimiento de obligaciones relacionados con el timbrado de la nómina, estos resultados 
se ven representados en nuestra tabla 7 a continuación.

Tabla 7. Comparación con Variable de Control

\begin{tabular}{cc}
\hline Variable & Prob \\
\hline X4*Xt & 0.1995
\end{tabular}

Fuente: Elaboración propia con datos obtenidos de Eviews.

Donde X4 representa la implementación de las TICS y Xt el número de trabajadores de las empresas encuestadas dentro de la investigación. Al llevar a cabo estas comparaciones se observa que el número de trabajadores de las empresas tienen relación con el factor de implementación de las TIC ya que aumenta considerablemente su significancia.

\section{CONCLUSIONES}

A partir del análisis de los datos obtenidos, se llega a las siguientes conclusiones:

La Implementación de las TIC resulta ser importante en relación a las variables de control en especial cuando el microempresario tiene más trabajadores a su cargo, sin embargo, como un factor que influye en el comportamiento tributario tiene menor representatividad de otros factores cuando los microempresarios no tienen empleados. Valorándose de manera parcial la Hipótesis establecida de que la implementación de las TIC ocasionó que los microempresarios se vieran afectados al tener que invertir en ellas a partir de la reforma fiscal del 2014. Con lo cual no se concuerda con lo establecido por Álvarez 2014 y González 2018 sobre la necesidad de implementar las TIC para el cumplimiento de las obligaciones fiscales.

Al llevar el análisis con el método Logit y las variables de control se comprobó que la implementación de las TIC, afecta más a las microempresas que tienen más empleados, ya que las obligaciones derivadas de la nómina están estrechamente relacionadas con el uso de las Tecnologías, como es el timbrado de la nómina, los pagos de las contribuciones de seguridad social, mismo que requieren de uso e implementación de las mismas, por lo cual se puede concluir que el número de empleados que tienen los microempresarios afecta directamente sobre el comportamiento tributario de los mismos. 
Vol. 7 Núm. 2 


\section{REFERENCIAS}

Álvarez Flores Lorena, Flores Reymundo Arturo. 2014. Mipymes; Necesidad de las TIC para cumplir las obligaciones fiscales, recuperado en: http://acacia.org.mx/busqueda/pdf/MIPYMES NECESIDAD DE LAS TIC PARA CUMP LIR OBLIGACIONES FISCALES.pdf

Arias Mayorga María Gabriela, Torres Mieles Mario Andrés. 2015. Análisis del comportamiento tributario de los contribuyentes del mercado de artículos varios Bolívar de la ciudad de Guayaquil en el periodo 2013-2014. ubicado en la parroquia

Salesiana.Recuperado de: https://dspace.ups.edu.ec/bitstream/123456789/9979/1/UPS-GT001102.pdf

Bedoya, Andrea y Vásconez, Byron. Entendiendo la moral tributaria en Ecuador. En: Fiscalidad. Revista Institucional del Servicio de Rentas Internas. Quito: SRI, (no. 05, julio a diciembre 2010): pp. 91-132.

Burga Argandoña Melissa Eleine.2014. Cultura tributaria y obligaciones tributarias en las empresas comerciales del emporio gamarra, repositorioacademico.usmp.edu.pe/bitstream/handle/usmp/1577/burga_ame.pdf;jsessionid=7 3FF8AC5A620C9F3D2CF10ABA7F8B8EC?sequence $=1$

Casar Maria Amparo. 2013. los mexicanos contra los impuestos https://www.nexos.com.mx/?p=15579

Castañeda Rodríguez Víctor Mauricio. 2015. La moral tributaria en América Latina y la corrupción como uno de sus determinantes. Revista Mexicana de Ciencias Políticas y Sociales. Universidad Nacional Autónoma de México

Nueva Época, Año LX, núm. 224 mayo-agosto de 2015

Gaibor, A., Manya, M., Piedrahita, D., y Carrera, W. (2010). Perfil socioeconómico del contribuyente de Guayaquil sujeto al control del Servicio de Rentas Internas en sus obligaciones tributarias.

González, D. (2006). Regímenes Especiales de Tributación para Pequeños

Contribuyentes en América Latina. Banco Interamericano de Desarrollo.

García Flores Ada Nely, González Padilla Mayra, Lazo Mirando Viky Melisa.2015.

Cultura tributaria de los contribuyentes bajo al Régimen General del Impuesto sobre la Renta.

García, PL. (2015). La desaparición del Régimen de Pequeños contribuyentes en la Reforma Fiscal de 2014: análisis y perspectivas. Documento de trabajo 630. Clasificación temática: Política Fiscal

Gómez, E. (2017). Nómina digital 2017. Colegio de Contadores Públicos Universidad de Guadalajara. Boletín Fiscal 137.

Hinojosa, A. V. (2013). La evasión y elusión fiscal: "su abordaje a través de la integración de una nueva estrategia educativa".

López J., Un sistema tributario complejo. España. Fecha de acceso: 10/08/2019. URL: http://www.notariado.org/liferay/c/document_library/get_file?folderId=12092\&name=DLFE55939.pdf

Lozano Rodríguez, E. (2014). Tributación de Pequeños Contribuyentes y Regímenes

Simplificados en Colombia. Revista de Derecho Privado, 1-14.

Moreno Neri J.J., Arellano Z. S.A., Obregon A. MM, Velasco A. Lizzette (2017). Consecuencias fiscales y económicas de la no deducibilidad de la nómina en contribuyentes del Régimen de Incorporación Fiscal de Ensenada, B. C. Compendio investigativo de la Academia Journals Celaya 2017. Recuperado en :

https://www.researchgate.net/publication/322554099_Consecuencias_fiscales_y_economicas _de_la_no_deducibilidad_de_la_nomina_en_contribuyentes_del_Regimen_de_Incorporacion _Fiscal_de_Ensenada_B_C

Obregón A. MM, Tamez G. Silverio. (2020). Factores del comportamiento tributario. Revista Vinculategica. Recuperado en: 
http://www.web.facpya.uanl.mx/Vinculategica/Vinculategica6_1/21\%20OBREGON_TAME Z.pdf 